\title{
Deep-Inspiration Breath-Hold PET/CT: Clinical Findings with a New Technique for Detection and Characterization of Thoracic Lesions
}

\author{
Gustavo S.P. Meirelles ${ }^{1}$, Yusuf Emre Erdi², Sadek A. Nehmeh², Olivia D. Squire ${ }^{1}$, Steven M. Larson ${ }^{1}$, \\ John L. Humm², and Heiko Schöder ${ }^{1}$ \\ ${ }^{1}$ Department of Radiology, Nuclear Medicine Service, Memorial Sloan-Kettering Cancer Center, New York, New York; and \\ ${ }^{2}$ Department of Medical Physics, Memorial Sloan-Kettering Cancer Center, New York, New York
}

\begin{abstract}
Respiratory motion during PET/CT acquisition can cause misregistration and inaccuracies in calculation of standardized uptake values (SUVs). Our aim was to compare the detection and characterization of thoracic lesions on PET/CT with and without a deep-inspiration protocol. Methods: We studied 15 patients with suspected pulmonary lesions who underwent clinical PET/ $\mathrm{CT}$, followed by deep-inspiration breath-hold (BH) PET/CT. In $\mathrm{BH}$ CT, the whole chest of the patient was scanned in $15 \mathrm{~s}$ at the end of deep inspiration. For BH PET, patients were asked to hold their breath 9 times for 20-s intervals. One radiologist reviewed images, aiming to detect and characterize pulmonary, nodal, and skeletal abnormalities. Clinical CT and BH CT were compared for number, size, and location of lesions. Lesion SUVs were compared between clinical PET and BH PET. Images were also visually assessed for accuracy of fusion and registration. Results: All patients had lesions on clinical $\mathrm{CT}$ and $\mathrm{BH}$ CT. Pulmonary BH CT detected more lesions than clinical CT in 13 of 15 patients (86.7\%). The total number of lung lesions detected increased from 53 with clinical CT to 82 with BH CT $(P<0.001)$. Eleven patients showed a total of 31 lesions with abnormal ${ }^{18} \mathrm{~F}-\mathrm{FDG}$ uptake. BH PET/CT had the advantage of reducing misregistration and permitted a better localization of sites with ${ }^{18}$ F-FDG uptake. A higher SUV was noted in 22 of 31 lesions on BH PET compared with clinical PET, with an average increase in SUV of 14\%. Conclusion: BH PET/CT enabled an increased detection and better characterization of thoracic lesions compared with a standard PET/CT protocol, in addition to more precise localization and quantification of the findings. The technique is easy to implement in clinical practice and requires only a minor increase in the examination time.
\end{abstract}

Key Words: PET/CT; breath-hold; imaging; ${ }^{18} \mathrm{~F}-\mathrm{FDG}$; cancer

J Nucl Med 2007; 48:712-719

DOI: 10.2967/jnumed.106.038034

\footnotetext{
Received Nov. 8, 2006; revision accepted Jan. 2, 2007.

For correspondence or reprints contact: Heiko Schöder, MD, Department of Radiology/Nuclear Medicine, Memorial Sloan-Kettering Cancer Center, 1275 York Ave., Box 77, New York, NY 10021.

E-mail: schoderh@mskcc.org
}

$\mathbf{T}$ he recent introduction in clinical routine of combined PET/CT scanners, which provide inline fusion of anatomic and functional images, has enabled a more precise localization characterization of sites with radiotracer uptake. This has led to an increased acceptance of the method as a clinical tool for the diagnosis, staging, restaging, and monitoring of patients with a wide variety of cancers (1-6).

Although the optimal protocol for PET/CT studies remains a subject of discussion, most centers perform the CT component of the examination in shallow breathing or at the end of expiration $(7,8)$. The PET component, because of its longer acquisition time, is performed during free tidal breathing. Therefore, even with an inline scanner, the coregistration of PET and CT can be imprecise, leading to misalignment of structures and lesions, repeated scans over certain regions, and omission of anatomic levels. Misalignment between PET and CT can reduce the diagnostic accuracy of PET/CT for lesion detection. The accuracy of PET/CT for treatment monitoring could also be limited by respiratory motion: Because the $\mathrm{CT}$ images are used for attenuation correction of PET emission data, misalignment between PET and CT lesions will lead to overestimation or underestimation of standardized uptake values (SUVs). Furthermore, respiratory motion causes a spread of tracer activity within a given lesion over a larger area and, thus, underestimation of the true activity concentration (9-11). Although experienced PET/CT readers are familiar with these limitations and consider them in their study interpretation (12), it would be desirable to eliminate these artifacts completely and develop an imaging technique that allows accurate PET/CT alignment in the thorax. Indeed, efforts in this regard have led to the development of four-dimensional (4D) PET/CT, whereby respiratory gating of PET and CT can reduce or even eliminate these artifacts (11). However, this technique has not gained wide acceptance in diagnostic imaging practice because of the long acquisition time for 4D PET/CT, time-consuming postprocessing of raw data, and a higher radiation dose delivered to the patient (11). 
Previous studies have shown the capability of acquiring PET/CT images at any phase within the respiratory cyclein a short period of time-equivalent to the one adopted and used in clinical practice with shallow breathing or at the end of expiration (9). The aim of this study is to compare the detection and characterization of thoracic lesions on regular clinical PET/CT with those on deepinspiration breath-hold (BH) PET/CT, acquired at a single breathing phase at end inspiration.

\section{MATERIALS AND METHODS}

\section{Patients}

Fifteen patients ( 8 male, 7 female; average age, 57.3 y; age range, 43-73 y) with a biopsy-proven diagnosis of cancer, confirmed by staff pathologists at our institution, were included in this prospective pilot study. Patients were selected for this study on the basis of the clinical referral, requesting evaluation of a documented or suspected primary or secondary malignancy in the lungs or mediastinum. Nine patients presented with newly diagnosed or recurrent/ metastatic lung cancer, 1 with newly diagnosed esophageal cancer, 3 with proven or suspected lung metastases from prior cervical or colorectal cancer, 1 with prostate-specific antigen (PSA) relapse and nodules in lungs and mediastinum that were later proven to be sarcoidosis, and 1 with rising thyroglobulin level after resection of thyroid cancer.

In addition to the clinical PET/CT examination, in which PET and CT images are acquired during shallow breathing, all patients underwent a deep-inspiration BH PET/CT scan. All patients gave written informed consent. The study protocol was approved by the institutional review board.

Patients fasted for at least $6 \mathrm{~h}$ before injection of 514-611 MBq of ${ }^{18}$ F-FDG. During the uptake phase of approximately $60 \mathrm{~min}$, patients remained in a quiet position and were trained to follow verbal breathing instructions ("breathe in, hold, relax"), which aimed to ensure that they could hold their breath for at least $20 \mathrm{~s}$ repeatedly.

\section{PET/CT Acquisition and Image Reconstruction}

All PET/CT studies were acquired on a Discovery LS PET/CT system (GE Healthcare). A scout image with settings of $30 \mathrm{~mA}$ and $120 \mathrm{kV}$ was first acquired to determine the scanning field of the patient. This was followed by helical CT using the following scanning parameters: $140 \mathrm{kV}, 80 \mathrm{~mA}$, a 5-mm-slice thickness in $4.25-\mathrm{mm}$ intervals, and gantry rotation period of $0.8 \mathrm{~s}$. This scan was then followed by a whole-body PET scan that covered the same area with $3 \mathrm{~min}$ per bed position. The effective axial field of view (FOV) was $14.8 \mathrm{~cm}$ for each bed position, as there was an overlapping of 5 slices between each axial FOV. These CT and PET scans were acquired under free shallow breathing, with no voice instructions.

After clinical PET/CT, patients remained in the same position (supine, arms above the head), and a plastic box with 2 infrared reflective markers was placed on the abdomen for respiratory motion tracking. The Real-Time Position Management (RPM) Respiratory Gating System (Varian Medical Systems) was used to monitor and track the patient's respiratory motion, using the amplitude-gating mode. A second chest CT, encompassing the entire lungs, was then obtained with identical parameters as in the first one, except for a voice command asking the patients to hold their breath in end-inspiration for the duration of the examination, which was approximately $15 \mathrm{~s}$.
PET data were then obtained for one $15-\mathrm{cm}$ bed position in 9 independent frames of $20 \mathrm{~s}$ each (total, $3 \mathrm{~min}$ ). During these 20-s acquisitions the patients were asked to hold their breath in endinspiration. Between each of the $9 \mathrm{BH}$ sessions, patients were given an approximately 20 -s period for rest and relaxation. Thus, the entire BH PET acquisition required on average 6 min per patient $(9 \times 20 \mathrm{~s}$ for acquisition $+8 \times 20 \mathrm{~s}$ for relaxation). In this pilot study, PET images were acquired for just one FOV to obtain proof-of-principle data and to limit patient discomfort. To ensure that PET images spatially match to CT images, the amplitude of the breathing signal displayed on the RPM monitor during PET was set to the same level as the amplitude of the BH CT.

At the end of the study, all of the $9 \times 20$-s sinogram frames were added to generate the final 3-min dataset, thus matching the clinical PET acquisition time. These data were then corrected for attenuation using the BH CT images and reconstructed with the clinical ordered-subsets expectation maximization (OSEM) reconstruction algorithm and parameters. The same reconstruction parameters were used for clinical PET/CT and BH PET/CT scans. The details of this new method were described recently (13).

\section{Image Analysis}

Clinical PET/CT and BH chest PET/CT studies were examined in separate instances by the same thoracic radiologist, who was unaware of the clinical indication for the study or other clinical data. The aim was to detect and characterize pulmonary, nodal, and skeletal abnormalities on clinical PET/CT and BH PET/CT studies.

Readings were performed on a dedicated workstation (Xeleris; GE Healthcare), which can display 3 orthogonal planes for CT, PET, and PET/CT fused images (sagittal, coronal, and transaxial) and a maximum-intensity-projection image.

The clinical CT was read first, followed by the BH CT of the same patient. The number, location, and characteristics of lesions were assessed on both CT examinations. The following CT windows were used: lung (window, 1,500 Hounsfield units [HU]; level, $-800 \mathrm{HU}$ ), soft tissue (window, $450 \mathrm{HU}$; level, $50 \mathrm{HU}$ ), and bone (window, 2,000 HU; level, $200 \mathrm{HU}$ ).

Pulmonary nodules were defined as rounded opacities surrounded by lung parenchyma, measuring up to $3 \mathrm{~cm}$; they were considered a mass if they were $>3 \mathrm{~cm}$. Consolidations and ground-glass opacities (GGOs) were defined as areas of increased lung attenuation that did or did not obscure the adjacent lung vessels, respectively. Cavities were defined as areas of reduced lung attenuation enclosed by walls (14).

Nodes were regarded as diseased when measuring $>1.0 \mathrm{~cm}$ in the short axis, except for some sites-such as the internal mammary chain or the diaphragmatic region, where any visualized node was considered pathologic. The lesion location was classified according to the mediastinal nodal station system (15).

After the interpretation of clinical CT and BH CT examinations, the same radiologist proceeded to analyze the clinical PET/CT and BH PET/CT. As BH PET images had been acquired for a single FOV, the analysis of clinical PET and clinical PET/CT scans was restricted to this one FOV of the BH PET.

The presence of ${ }^{18} \mathrm{~F}$-FDG uptake in lesions detected on CT was assessed with the correlation of CT, PET (corrected and uncorrected), and PET/CT fusion images. ${ }^{18} \mathrm{~F}-\mathrm{FDG}$ uptake was considered positive if it was greater than that in the surrounding normal tissue. A circular region of interest (ROI) was drawn on each lesion with ${ }^{18}$ F-FDG uptake, on transaxial images, encompassing 
the entire lesion. The maximum SUV (SUVmax), normalized to body weight, was recorded for each finding.

A comparison was performed between the clinical CT and the $\mathrm{BH}$ CT for the number, size, and location of lesions. The ${ }^{18} \mathrm{~F}-\mathrm{FDG}$ PET SUVmax values were compared between clinical PET and BH PET. Images were also visually assessed on clinical PET/CT and BH PET/CT for accuracy of fusion and alignment. The number of lesions per patient on clinical CT versus BH CT and the SUVs on clinical PET versus BH PET were compared using a paired $t$ test. A $P$ value $<0.05$ was considered significant.

\section{RESULTS}

\section{CT Findings}

All patients had thoracic lesions (pulmonary, nodal, or skeletal) on clinical CT and BH CT (Table 1). The most frequent were pulmonary, followed by nodal and skeletal lesions. Compared with clinical CT, the BH CT detected more pulmonary lesions in 13 of the 15 patients (87\%). No additional lesions were seen with BH CT in lymph nodes or in the skeleton.

BH CT detected 29 additional lung lesions in 13 patients, especially nodules smaller than $0.5 \mathrm{~cm}(P<0.001$; Fig. 1$)$. Nodules and GGOs were most frequently depicted with the BH CT. Tables 2 and 3 show the number, characteristics, and distribution of the pulmonary lesions detected by both CT modalities.

The BH CT allowed more precise localization and characterization of pulmonary findings than clinical CT in all cases. Nodules, GGOs, and consolidations were better defined and showed sharper contours. In 1 patient with diffuse disease, use of the $\mathrm{BH}$ technique permitted an improved distinction between normal and diseased areas and enhanced the definition of disease boundaries (Fig. 2). In 1 case of lung cancer, use of the $\mathrm{BH}$ technique was particularly useful because of the detection of signs of pulmonary carcinomatous lymphangitis on the $\mathrm{BH}$ CT in a patient without evidence of distant disease on clinical PET/CT. These findings were characterized by irregular septal thickening, GGOs, and nodules, which were not seen on the clinical CT (Fig. 3). This patient also had foci of centrilobular emphysema on the upper lobes, detected only on the BH CT.

Twenty-seven lung lesions were seen on clinical CT and BH CT and were measured on both modalities. The size of

TABLE 1

Pulmonary, Nodal, and Skeletal Chest Lesions Detected on Clinical CT (CCT) and BH CT

\begin{tabular}{|c|c|c|c|c|}
\hline $\begin{array}{l}\text { Location } \\
\text { of lesions }\end{array}$ & $\begin{array}{c}\text { No. of } \\
\text { patients } \\
\text { with lesions } \\
\text { on CCT }\end{array}$ & $\begin{array}{l}\text { No. of } \\
\text { lesions } \\
\text { on CCT }\end{array}$ & $\begin{array}{c}\text { No. of } \\
\text { patients } \\
\text { with lesions } \\
\text { on BH CT }\end{array}$ & $\begin{array}{l}\text { No. of } \\
\text { lesions on } \\
\text { BH CT }\end{array}$ \\
\hline Lung & 15 & 33 & 15 & 62 \\
\hline Lymph nodes & 4 & 12 & 4 & 12 \\
\hline Bones & 2 & 8 & 2 & 8 \\
\hline Total & & 53 & & 82 \\
\hline
\end{tabular}
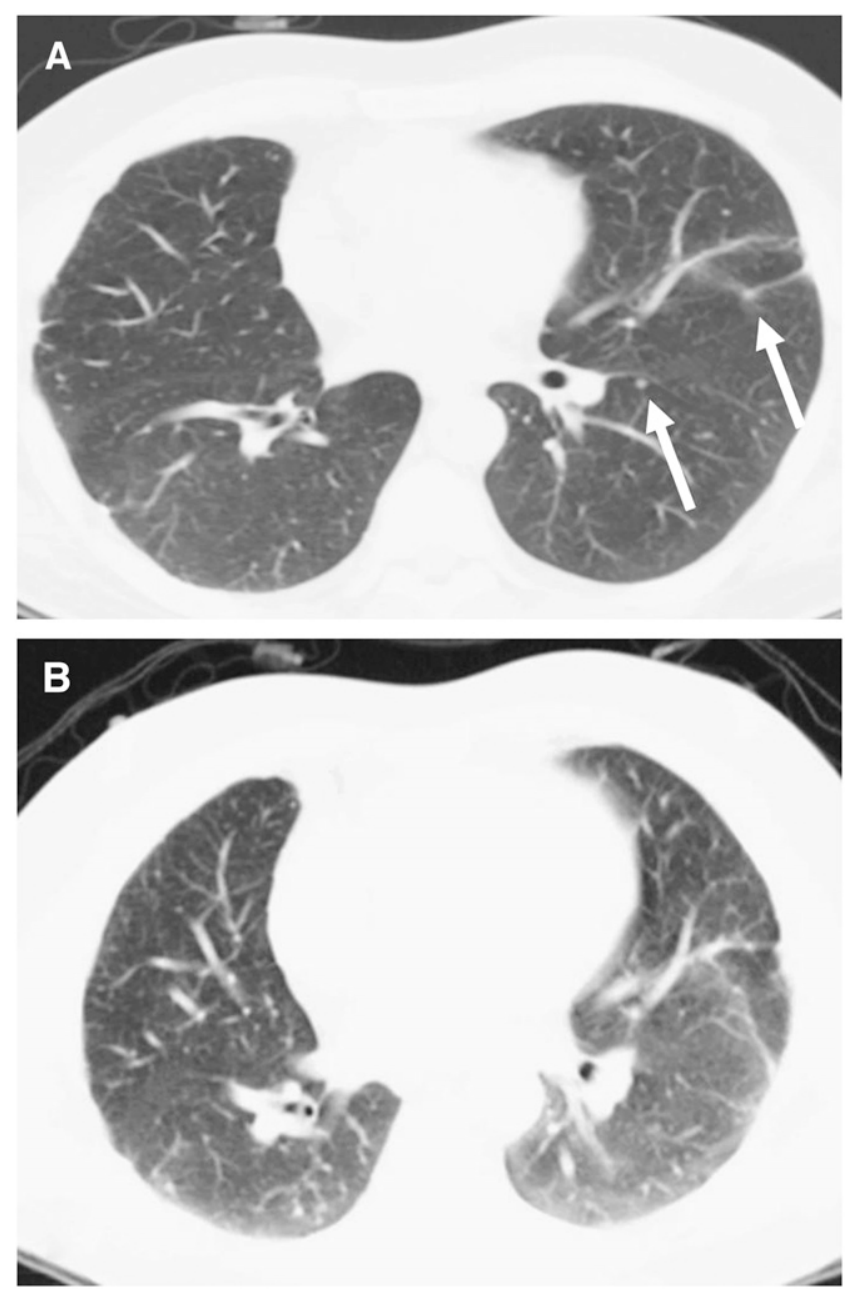

FIGURE 1. (A) Patient with lung cancer with 2 additional nodules on left lung on BH CT (arrows). (B) Clinical CT does not show lesions.

all lesions detected on clinical CT ranged from 0.3 to $9.8 \mathrm{~cm}$ (average $\pm \mathrm{SD}, 1.5 \pm 2.2 \mathrm{~cm}$ ) and on BH CT ranged from 0.2 to $9.6 \mathrm{~cm}$ (average $\pm \mathrm{SD}, 1.0 \pm 1.7 \mathrm{~cm}$ ). With specific regard to lung nodules, the average size for nodules detected on clinical CT was $0.9 \mathrm{~cm}$ and on BH CT it was $0.7 \mathrm{~cm}$. No patient had lesions detected exclusively with clinical CT.

Four patients had a total of 12 enlarged lymph nodes on clinical CT and BH CT. Eight of these nodes were located

TABLE 2

Pulmonary Lesions Detected on Clinical CT (CCT) and $\mathrm{BH}$ CT

\begin{tabular}{lrc}
\hline Type of lesion & CCT & BH CT \\
\hline Nodules & 22 & 49 \\
Masses & 4 & 4 \\
Consolidations & 4 & 4 \\
GGOs & 2 & 4 \\
Cavities & 1 & 1 \\
Total & 33 & 62 \\
\hline
\end{tabular}


TABLE 3

Location of Pulmonary Lesions Detected on Clinical CT (CCT) and $\mathrm{BH} \mathrm{CT}$

\begin{tabular}{lrc}
\hline Location of lesions & CCT & BH CT \\
\hline Right upper lobe & 14 & 20 \\
Right middle lobe & 3 & 8 \\
Right lower lobe & 10 & 12 \\
Left upper lobe & 5 & 10 \\
Lingula & 1 & 2 \\
Left lower lobe & 5 & 7
\end{tabular}

Some lesions extended beyond interlobar fissures and, thus, were noted in 2 adjacent pulmonary lobes. Therefore, the number of lesions listed here is larger than the total number of lesions detected, which is shown in Table 2.

in the mediastinum (sites 2R, 4R, 7, and 8), 3 in the pulmonary hila, and 1 in the right internal mammary chain. No additional nodal lesions were seen on BH CT.

Three patients had pleural effusions and 1 patient had a pericardial effusion on both clinical CT and BH CT. No additional effusions were detected on BH CT.

Two patients had bone lesions on CT, for a total of 8 findings, located in the ribs $(n=4)$, scapulae $(n=2)$, spine $(n=1)$, and right clavicle $(n=1)$. The BH CT did not detect additional skeletal abnormalities.

\section{PET and PET/CT Findings}

Eleven of the 15 patients had sites of abnormal ${ }^{18} \mathrm{~F}-\mathrm{FDG}$ uptake on the PET component of the examination. In the remaining 4 patients, no ${ }^{18} \mathrm{~F}$-FDG uptake was noted in the lesions detected on the $\mathrm{CT}(n=3)$ or the lesion was not included in the BH PET FOV $(n=1)$.

Thirty-nine of the 53 lesions noted on clinical CT showed some ${ }^{18}$ F-FDG uptake on clinical PET. Thirty-one of these
39 lesions were visualized on both clinical PET and BH PET (13 in lung, 11 in lymph nodes, and 7 in skeleton). The remaining 8 lesions were outside the BH PET FOV and, therefore, are not included in the following analysis. Accordingly, Table 4 shows the SUV measurements only for those ${ }^{18} \mathrm{~F}-\mathrm{FDG}$-avid lesions that were included in the imaged FOV on both clinical PET and BH PET. In $75 \%$ of the findings, the SUVmax values were higher on BH PET when compared with clinical PET $(P<0.01$; Fig. 4 and Table 4). The largest absolute increase occurred from SUV 7.8 on clinical PET to SUV 12.0 on BH PET.

The smallest change between SUVmax from clinical PET versus BH PET was noted for bone lesions. Here, the impact of respiratory motion depends on the lesion location and may be negligible for lesions in vertebral bodies or posterior ribs.

$\mathrm{BH} \mathrm{PET} / \mathrm{CT}$ had the advantage of reducing misregistration, as both the CT and the PET component were acquired in the same respiratory phase. This was particularly useful for distinguishing between pulmonary and extrapulmonary lesions in the chest wall or mediastinum. In 1 patient, focal ${ }^{18}$ F-FDG uptake localized to the lung on clinical PET/CT could be proven to represent a rib metastasis on BH PET/CT (Fig. 5). One small nodule that was not seen on clinical PET/ CT showed mild ${ }^{18} \mathrm{~F}-\mathrm{FDG}$ uptake on BH PET/CT; this was an additional site of metastasis in patient with lymphangitis.

Pleural or pericardial effusions showed no ${ }^{18} \mathrm{~F}-\mathrm{FDG}$ uptake on either clinical PET or BH PET. The same was true for lung cavities and areas of GGOs.

\section{DISCUSSION}

This study demonstrates that the new technique of deepinspiration $\mathrm{BH} \mathrm{PET} / \mathrm{CT}$ is superior to standard clinical $\mathrm{PET} / \mathrm{CT}$ for the detection and characterization of thoracic
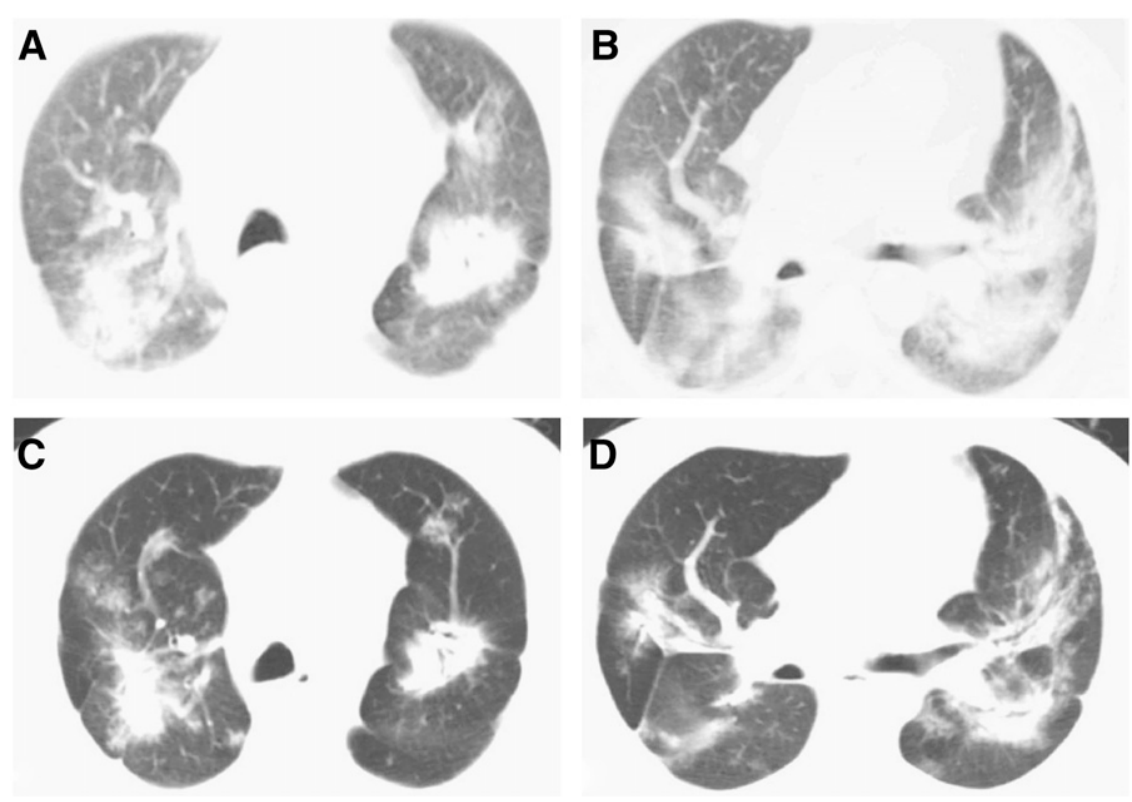

FIGURE 2. Patient with widespread metastatic colonic adenocarcinoma to lungs, with consolidations and GGOs. Although clinical CT demonstrates findings ( $\mathrm{A}$ and $\mathrm{B}), \mathrm{BH}$ examination $(\mathrm{C}$ and $\mathrm{D})$ has much better quality to define lesions' borders and characteristics. 

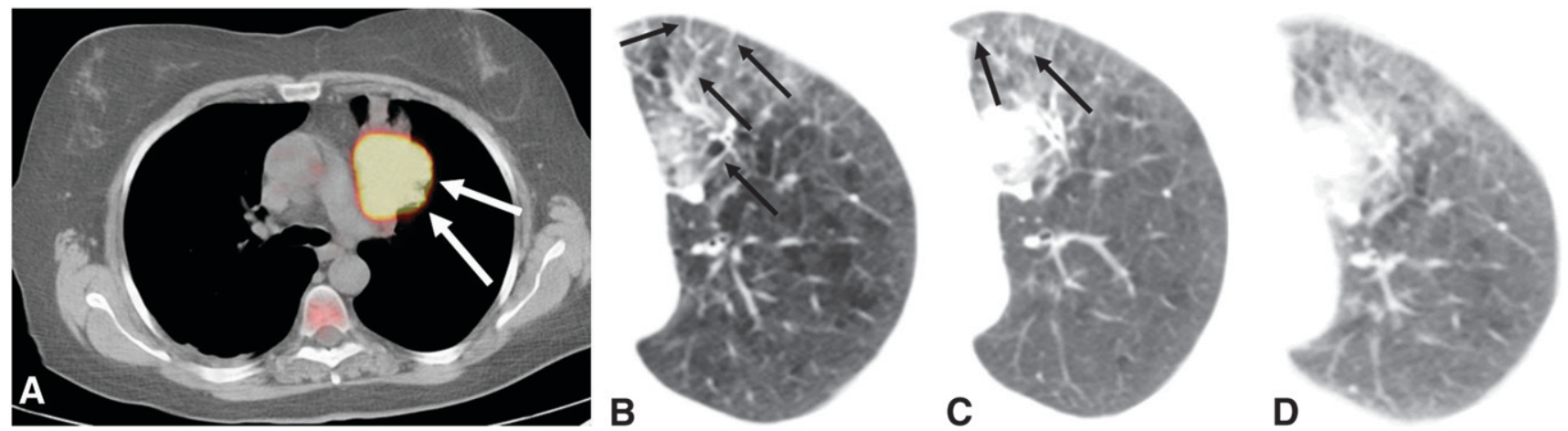

FIGURE 3. (A) BH PET/CT of patient with adenocarcinoma of left lung, with ${ }^{18} \mathrm{~F}-\mathrm{FDG}$ PET uptake in pulmonary mass (arrows) and SUVmax of $23.3 \mathrm{~g} / \mathrm{mL}$, which was $21.2 \mathrm{~g} / \mathrm{mL}$ on clinical PET/CT. (B and C) Use of BH CT allows detection of carcinomatous lymphangitis adjacent to tumor, characterized by septal thickening (B, arrows), GGOs, and small nodules (C, arrows). (D) It is very hard to depict and characterize the same findings on clinical non-BH CT.

lesions. When compared with clinical scans, the $\mathrm{BH}$ method permitted detection of more pulmonary lesions on CT in $87 \%$ of patients. It also reduced breathing-induced artifacts, which would otherwise lead to misregistration of images on the fused dataset, inaccuracies in SUV measurements, and erroneous localization of lung or chest wall lesions.

The use of BH PET/CT enabled a better localization and definition of the lesions, especially in the lungs, when compared with the regular clinical PET/CT protocol. In 1 case, the clinical stage of a patient with lung cancer was changed on BH PET/CT, because of a clearer visualization on CT of findings compatible with carcinomatous lymphangitis in the lung.

A major advantage of the BH PET/CT technique is the precise registration of fused PET/CT images. Although the experienced PET reader may often account for misregistration artifacts, in some cases this misalignment can pose a serious diagnostic problem $(12,16,17)$. This is exemplified in 1 of our patients with a chest lesion that was incorrectly localized to the right lung on clinical images but was proven to be located in a rib using the $\mathrm{BH}$ technique.

Previously, shallow expiration has been recommended as a method to minimize motion artifacts. However, although shallow breathing can minimize the lung motion, it does not eliminate it completely. This can be achieved only in a clinically practical manner with our proposed BH PET/CT technique, which eliminates all motion artifacts.

In cancer patients, PET and PET/CT are increasingly used in evaluation of the response to therapy. For this intent, most physicians rely on changes in SUV measurements from serial PET studies over time $(18,19)$. However, as images acquired during free breathing are prone to respiratory artifacts, with blurring of lesions and mismatch between PET and CT data, inaccuracies in PET measures of SUV may propagate into undesirable overestimation or underestimation of the real treatment response $(9,16)$. Adoption of the $\mathrm{BH}$ technique enables a more precise measurement of

TABLE 4

Behavior of SUVmax on Clinical PET and BH PET

\begin{tabular}{|c|c|c|c|c|c|}
\hline Lesions & $\begin{array}{l}\text { Clinical PET } \\
\text { SUVmax* }\end{array}$ & $\begin{array}{l}\text { BH PET } \\
\text { SUVmax* }\end{array}$ & \multicolumn{2}{|c|}{$\begin{array}{l}\text { SUVmax on BH PET } \\
\text { vs. clinical PET }\end{array}$} & $\begin{array}{l}\% \text { difference in } \\
\text { SUVmax on BH PET } \\
\text { vs. clinical PET }\end{array}$ \\
\hline All $(n=31)$ & $6.0(1.3-22.2)$ & $6.8(1.5-22.3)$ & $\begin{array}{l}\text { Increase } \\
\text { Decrease } \\
\text { Same }\end{array}$ & $\begin{array}{r}22 \\
6 \\
3\end{array}$ & $\begin{array}{c}\uparrow 14 \\
(\downarrow 16-\uparrow 73)\end{array}$ \\
\hline Lung $(n=13)$ & $7.3(1.3-22.2)$ & $8.0(1.5-22.3)$ & $\begin{array}{l}\text { Increase } \\
\text { Decrease } \\
\text { Same }\end{array}$ & $\begin{array}{r}11 \\
1 \\
1\end{array}$ & $\begin{array}{c}\uparrow 11 \\
(\downarrow 16-\uparrow 32)\end{array}$ \\
\hline Nodal $(n=11)$ & $4.9(1.9-10.2)$ & $6.1(1.6-12.1)$ & $\begin{array}{l}\text { Increase } \\
\text { Decrease } \\
\text { Same }\end{array}$ & $\begin{array}{l}7 \\
3 \\
1\end{array}$ & $\begin{array}{c}\uparrow 23 \\
(\downarrow 7-\uparrow 73)\end{array}$ \\
\hline Bone $(n=7)$ & $5.2(2.4-10.0)$ & $5.5(2.5-10.3)$ & $\begin{array}{l}\text { Increase } \\
\text { Decrease } \\
\text { Same }\end{array}$ & $\begin{array}{l}4 \\
2 \\
1\end{array}$ & $\begin{array}{c}\uparrow 7 \\
(\downarrow 4-\uparrow 31)\end{array}$ \\
\hline
\end{tabular}



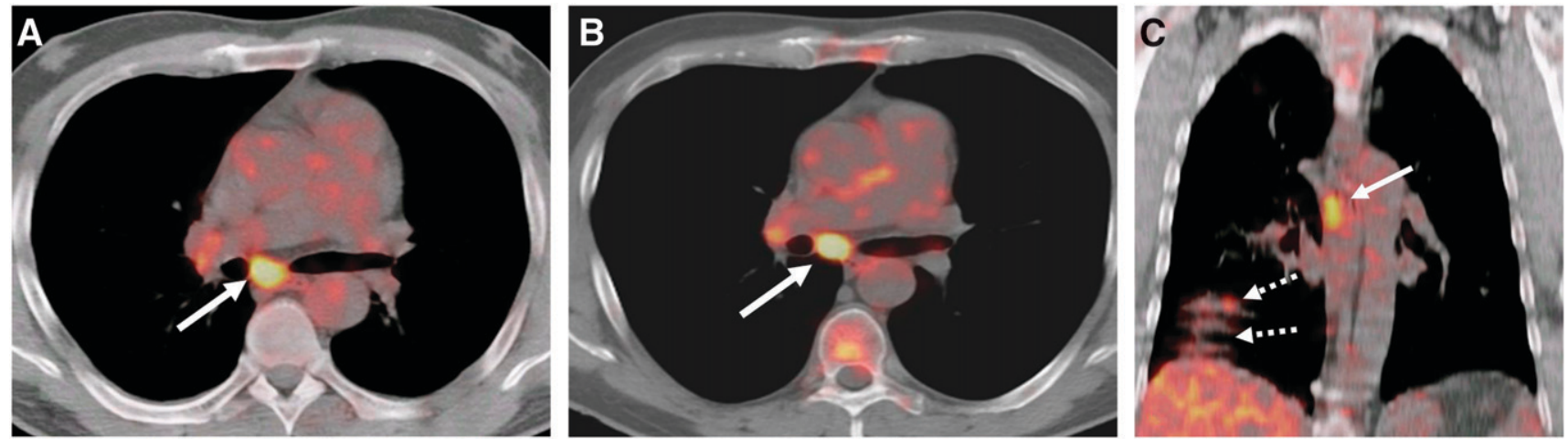

FIGURE 4. Lung cancer metastasis to subcarinal (level 7) lymph node. Although clinical PET/CT detects lesion (A, arrow), SUVmax of $7.8 \mathrm{mg} / \mathrm{dL}$ is significantly lower than SUVmax of $12.0 \mathrm{mg} / \mathrm{dL}$ measured on same lesion with BH PET/CT (B, arrow). (C) Coronal view of clinical PET/CT reveals breathing artifacts near diaphragm (dashed arrows) in addition to subcarinal node (solid arrow).

SUVs, and SUV measures obtained from BH images of lung lesions are expected to be higher than those from non-BH images $(9,11)$. In our study, SUV numbers calculated on BH PET were up to $73 \%$ higher than those on clinical PET. Such an increase in SUV is more pronounced in lesions susceptible to breathing motion, including small lung nodules or lymph nodes in mobile sites, such as the pulmonary hila. Indeed, the vast majority of lung and hilar lesions showed an increase in SUVmax on the BH PET images. On the other hand, in some lesions, the SUV on the BH images was lower than that on clinical PET. This included one small lung lesion with SUVmax of 1.6 compared with 1.9. However, it should be noted that SUV numbers below 2.5 are more prone to inaccuracies, as the lesion contrast is low and image noise increases the uncertainty in SUV calculation. In another 5 lesions, the SUV numbers on BH PET were also slightly lower than those on the clinical study, but these differences were between $4 \%$ and $7 \%$ and, thus, within the limits of reproducibility for SUV measurements in general (18). However, more commonly, increases in SUV were observed on BH PET images, exceeding 10\% in 15 of the total of 31 lesions in our study. Indeed, increases in SUV are the expected consequence of $\mathrm{BH}$ imaging in lesions with significant motion artifacts. In this pilot study, we did not observe any SUV change that would have resulted in designating a given lesion malignant instead of benign (or vice versa) if certain cut-offs, such as SUV 2.5 or 3.0, were to be used in characterizing lung nodules. This question should be addressed in future investigations. Lesions in vertebral bodies and large lesions in the lung parenchyma or large lymph nodes may show less movement with respiration and, accordingly, changes in the SUV may not be so remarkable.

We did not observe any obvious differences in clinical stage between clinical PET and BH PET. However, we were able to characterize the pattern of carcinomatous lymphangitis in one case on BH CT, which would not have been possible on clinical CT.

In contrast to 4D PET/CT, where data are acquired in several breathing phases (11), deep-inspiration BH PET/CT uses data from only a single respiratory phase, which considerably shortens the acquisition time from about 10 to 3 min per bed position. In addition, during 4D PET/CT,
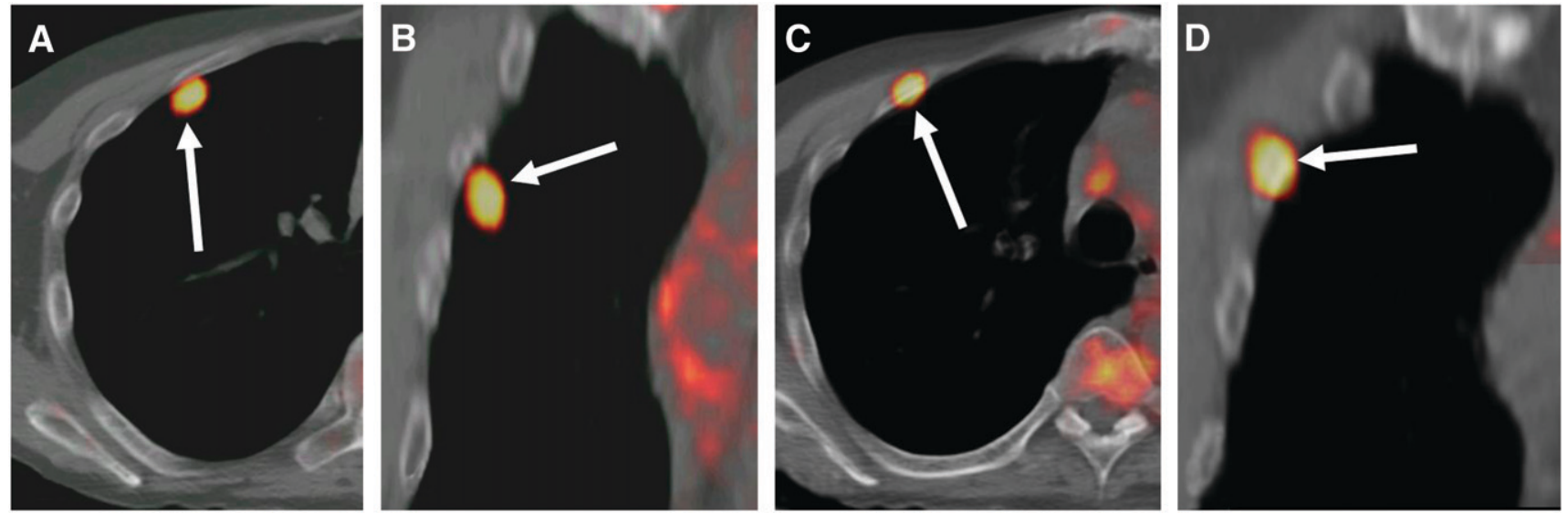

FIGURE 5. (A and B) Clinical PET/CT showed focus of ${ }^{18} \mathrm{~F}-\mathrm{FDG}$ uptake projecting on upper lobe of right lung on transaxial (A) and coronal (B) images (arrows). (C and D) BH PET/CT clearly localized focus of ${ }^{18} \mathrm{~F}-\mathrm{FDG}$ uptake to right third rib on transaxial (C) and coronal (D) images (arrows). This is clear example of mislocalization on standard PET/CT without BH technique. 
the patient must maintain a regular breathing pattern for an extended period of time, which is often not feasible in individuals with underlying lung disease. Here we have shown that in clinical practice the use of a single breathing phase in BH PET/CT is sufficient, so that the acquisition time is equal to that in standard clinical PET protocols. In most patients, the entire lung can be imaged with 2 axial FOVs $(2 \times 15 \mathrm{~cm})$ during BH PET. The acquisition time for each FOV is $6 \mathrm{~min}$, so that additional imaging time for the entire lung is about $12 \mathrm{~min}$. The additional imaging time for BH CT was negligible. The time for postprocessing of the images was also negligible, because both PET and CT images are acquired on the basis of the standard clinical PET/CT protocol. There is no additional radiation dose for the patient from the BH PET scan. The additional estimated effective dose from the BH CT scan is about $4.2 \mathrm{mSv}$, but introduced in clinical practice it would be expected that the $\mathrm{BH}$ CT of the lungs replaces the current low-dose CT of the lungs in shallow breathing. The eventual radiation dose using this technique, therefore, may not be much greater than that in current practice, but more accurate information could be obtained.

This new technique is straightforward and could be introduced in clinical practice easily, but patient cooperation is critical for the success of the study. Note that, although all 15 patients included were primarily referred for staging or follow-up of cancer, none of them had any problems following the breathing instructions. From a technical point of view, it is also quite simple to perform $\mathrm{BH}$ studies. A PET/CT technologist can perform this study with minimal training. The only additional charge is to observe the patient's breathing pattern on the monitor and to instruct the patient to hold his or her breath for $20 \mathrm{~s}$ and to relax again. For this purpose we have used a dedicated RPM device. However, any other respiratory motion-tracking system that enables display of the breathing amplitude could likely also be used. The current study was designed for an $\sim 15-\mathrm{cm}$ single PET FOV. Covering the entire lung ( $\sim 30 \mathrm{~cm}$ axially) would generally require 2 bed positions and, therefore, approximately $20 \mathrm{BHs}$. It is not known whether a sequence of $20 \mathrm{BHs}$ might be difficult for some patients. However, our previous experience in respiratory gating suggests that an acquisition time of $1 \mathrm{~min}$ per FOV may be sufficient to evaluate changes in SUV of lung lesions caused by breathing motion (11). Hence, if 2 FOVs are to be acquired, it may be possible to reduce the acquisition time to $5 \times 20-\mathrm{s} \mathrm{BH}$ periods per FOV, and a total of $10 \mathrm{BH}$ periods of $20 \mathrm{~s}$ each for the entire lung.

This work was a pilot study with a small number of patients. One limitation in our study was the absence of pathologic confirmation of the suspected thoracic lesions and the lack of subsequent examinations, which we hope to address in future investigations. The clinical significance of additional lesions detected with BH PET/CT is currently unclear, as most of them correspond to pulmonary micronodules for which there is currently no standardized man- agement $(20,21)$. The potential effect of BH PET/CT on assigning a clinical stage of disease also needs further study. Finally, the clinical significance of differences in SUV measurements between clinical PET/CT and BH PET/ $\mathrm{CT}$, especially in conjunction with response assessment, needs to be addressed in future studies. After treatment with chemotherapy or radiation therapy, ${ }^{18} \mathrm{~F}$-FDG uptake in cancerous lesions is expected to decline. In addition to standardizing other factors-such as the time interval between tracer injection, acquisition, and reconstruction parameters (22) — for lung lesions it would be important to ensure that changes in SUV do in fact represent a true finding rather than a technical artifact related to differences in breathing motion between the 2 scans. With PET/CT in particular, Erdi et al. have shown that SUV measures are affected by the degree of mismatch between PET and CT datasets (16). In the pre- and posttreatment setting, the CT of the combined PET/CT may be acquired at different breathing phases, which by itself might result in different SUV numbers. Such mismatches between PET and CT are essentially unavoidable with current acquisition techniques but could be eliminated effectively with the proposed $\mathrm{BH}$ method.

\section{CONCLUSION}

Our results demonstrate an increased detection and better characterization of lung lesions with BH PET/CT compared with a standard clinical PET/CT protocol. The technique is easy to implement in clinical practice and requires only a minor increase in the examination time. The use of deepinspiration BH PET/CT enabled a more precise localization of chest lesions in addition to reducing breathing artifacts, misregistration, and inaccuracies in SUV measurements. As a consequence, more precise coregistrations between PET and CT images and accurate quantification of ${ }^{18} \mathrm{~F}-\mathrm{FDG}$ activity concentrations in lung lesions can be obtained, the clinical benefits of which will be addressed in future studies.

\section{REFERENCES}

1. Antoch G, Saoudi N, Kuehl H, et al. Accuracy of whole-body dual-modality fluorine-18-2-fluoro-2-deoxy-D-glucose positron emission tomography and computed tomography (FDG-PET/CT) for tumor staging in solid tumors: comparison with CT and PET. J Clin Oncol. 2004;22:4357-4368.

2. Lardinois D, Weder W, Hany TF, et al. Staging of non-small-cell lung cancer with integrated positron-emission tomography and computed tomography. N Engl J Med. 2003;348:2500-2507.

3. Schöder H, Larson SM, Yeung HW. PET/CT in oncology: integration into clinical management of lymphoma, melanoma, and gastrointestinal malignancies. J Nucl Med. 2004;45(suppl 1):72S-81S.

4. Schöder H, Yeung HW, Gonen M, Kraus D, Larson SM. Head and neck cancer: clinical usefulness and accuracy of PET/CT image fusion. Radiology. 2004;231: $65-72$.

5. von Schulthess GK. Positron emission tomography versus positron emission tomography/computed tomography: from "unclear" to "new-clear" medicine. Mol Imaging Biol. 2004;6:183-187.

6. von Schulthess GK, Steinert HC, Hany TF. Integrated PET/CT: current applications and future directions. Radiology. 2006;238:405-422. 
7. Beyer T, Antoch G, Muller S, et al. Acquisition protocol considerations for combined PET/CT imaging. J Nucl Med. 2004;45(suppl 1):25S-35S.

8. Blodgett TM, McCook BM, Federle MP. Positron emission tomography/computed tomography: protocol issues and options. Semin Nucl Med. 2006;36:157-168.

9. Nehmeh SA, Erdi YE, Ling CC, et al. Effect of respiratory gating on quantifying PET images of lung cancer. $J$ Nucl Med. 2002;43:876-881.

10. Nehmeh SA, Erdi YE, Ling CC, et al. Effect of respiratory gating on reducing lung motion artifacts in PET imaging of lung cancer. Med Phys. 2002;29: 366-371.

11. Nehmeh SA, Erdi YE, Pan T, et al. Four-dimensional (4D) PET/CT imaging of the thorax. Med Phys. 2004;31:3179-3186.

12. Sarikaya I, Yeung HW, Erdi Y, Larson SM. Respiratory artefact causing malpositioning of liver dome lesion in right lower lung. Clin Nucl Med. 2003; 28:943-944.

13. Nehmeh S, Erdi Y, Meirelles G, et al. Deep-inspiration-breath-hold (DIBH) PET/ CT imaging of the thorax. J Nucl Med. 2007;48:22-46.

14. Austin JH, Muller NL, Friedman PJ, et al. Glossary of terms for CT of the lungs: recommendations of the Nomenclature Committee of the Fleischner Society. Radiology. 1996;200:327-331.

15. Mountain CF, Dresler CM. Regional lymph node classification for lung cancer staging. Chest. 1997;111:1718-1723.
16. Erdi YE, Nehmeh SA, Pan T, et al. The CT motion quantitation of lung lesions and its impact on PET-measured SUVs. J Nucl Med. 2004;45:12871292.

17. Osman MM, Cohade C, Nakamoto Y, Marshall LT, Leal JP, Wahl RL. Clinically significant inaccurate localization of lesions with PET/CT: frequency in 300 patients. J Nucl Med. 2003;44:240-243.

18. Weber WA, Ziegler SI, Thodtmann R, Hanauske AR, Schwaiger M. Reproducibility of metabolic measurements in malignant tumors using FDG PET. J Nucl Med. 1999;40:1771-1777.

19. Young H, Baum R, Cremerius U, et al. Measurement of clinical and subclinical tumour response using $\left[{ }^{18} \mathrm{~F}\right]$-fluorodeoxyglucose and positron emission tomography: review and 1999 EORTC recommendations-European Organization for Research and Treatment of Cancer (EORTC) PET Study Group. Eur J Cancer. 1999;35:1773-1782.

20. Benjamin MS, Drucker EA, McLoud TC, Shepard JA. Small pulmonary nodules: detection at chest CT and outcome. Radiology. 2003;226:489-493.

21. Swensen SJ, Jett JR, Hartman TE, et al. CT screening for lung cancer: five-year prospective experience. Radiology. 2005;235:259-265.

22. Schöder H, Erdi YE, Chao K, Gonen M, Larson SM, Yeung HW. Clinical implications of different image reconstruction parameters for interpretation of whole-body PET studies in cancer patients. J Nucl Med. 2004;45:559-566. 